\title{
Bone morphogenetic protein signaling as novel therapeutic target in pheochromocytoma
}

\author{
Andrea Richter ${ }^{1}$, Ines Leinhäuser1,2, Misu Lee1, Ines Höfig'2, Natasa Anastasov², Falko Fend ${ }^{3}$, Tonino Ercolino \\ Massimo Mannelli5, Anne-Paule Gimenez-Roqueplo6, Mercedes Robledo7, Ronald R. de Krijger ${ }^{8}$, Felix Beuschlein ${ }^{9}$, \\ Michael J. Atkinson ${ }^{2}$ and Natalia S. Pellegata ${ }^{1}$
}

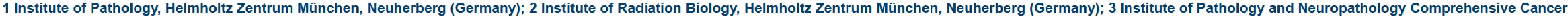

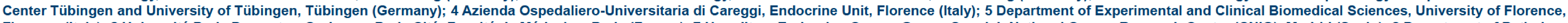

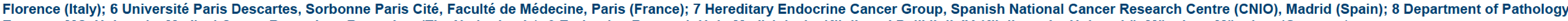
Erasmus MC University Medial Center Roterdam, Roterdam (T, Fa Ner

\section{Background}

MENX is a spontaneous multiple endocrine neoplasia-like syndrome in the rat, which is caused by a biallelic germline mutation in the Cdkn1b gene, encoding a very unstable mutant p27 protein (Figure 1C) [1]. MENX predisposes, among other neoplasias, to the development of bilateral pheochromocytomas (PCC) with complete penetrance (Figure 1) [2]. Gene expression profiling of MENX rat PCCs identified the growth factor Bmp7 (bone morphogenetic protein 7) as highly expressed in tumors versus normal adrenal medulla (Figure 1D) [3]. Previous work demonstrated that upregulation of Bmp7 enhances proliferation, migration and invasion of PCC cells. In primary rat PCC cells Bmp7 expression sustained cell viability. In PCC Bmp7 signals through the PI3K/AKT/mTOR pathway and integrin $\beta 1$ [3]. The small molecule antagonist DMH1 is a second-generation analog of dorsomorphin and inhibits most selectively BMP type I receptors (BMPR-I) (Figure 2) [4].
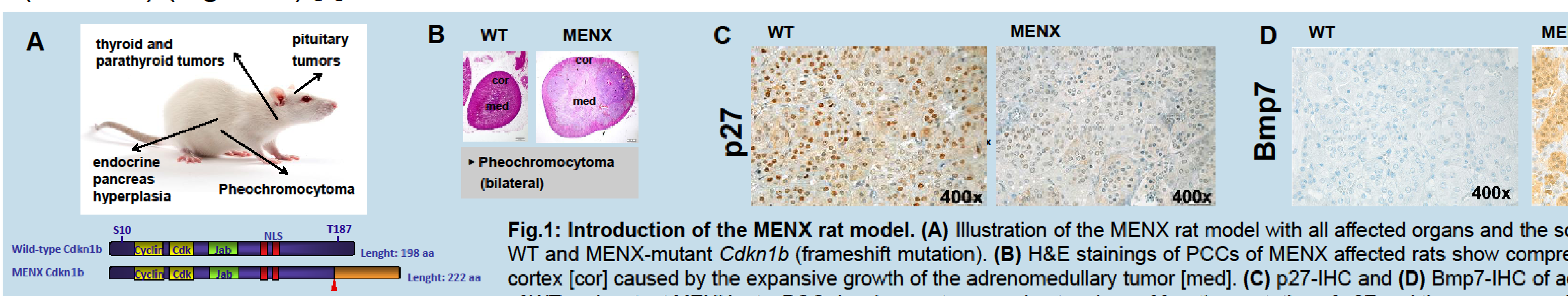

Fig.1: Introduction of the MENX rat model. (A) Illustration of the MENX rat model with all affected organs and the schematic structure of WT and MENX-mutant Cdkn1b (frameshift mutation). (B) H\&E stainings of PCCs of MENX affected rats show compression of the adrenal cortex [cor] caused by the expansive growth of the adrenomedullary tumor [med]. (C) p27-IHC and (D) Bmp7-IHC of adrenomedullary cells of WT and mutant MENX rats. PCC development occurs due to a loss of function mutation of p27 and the overexpression of Bmp7.

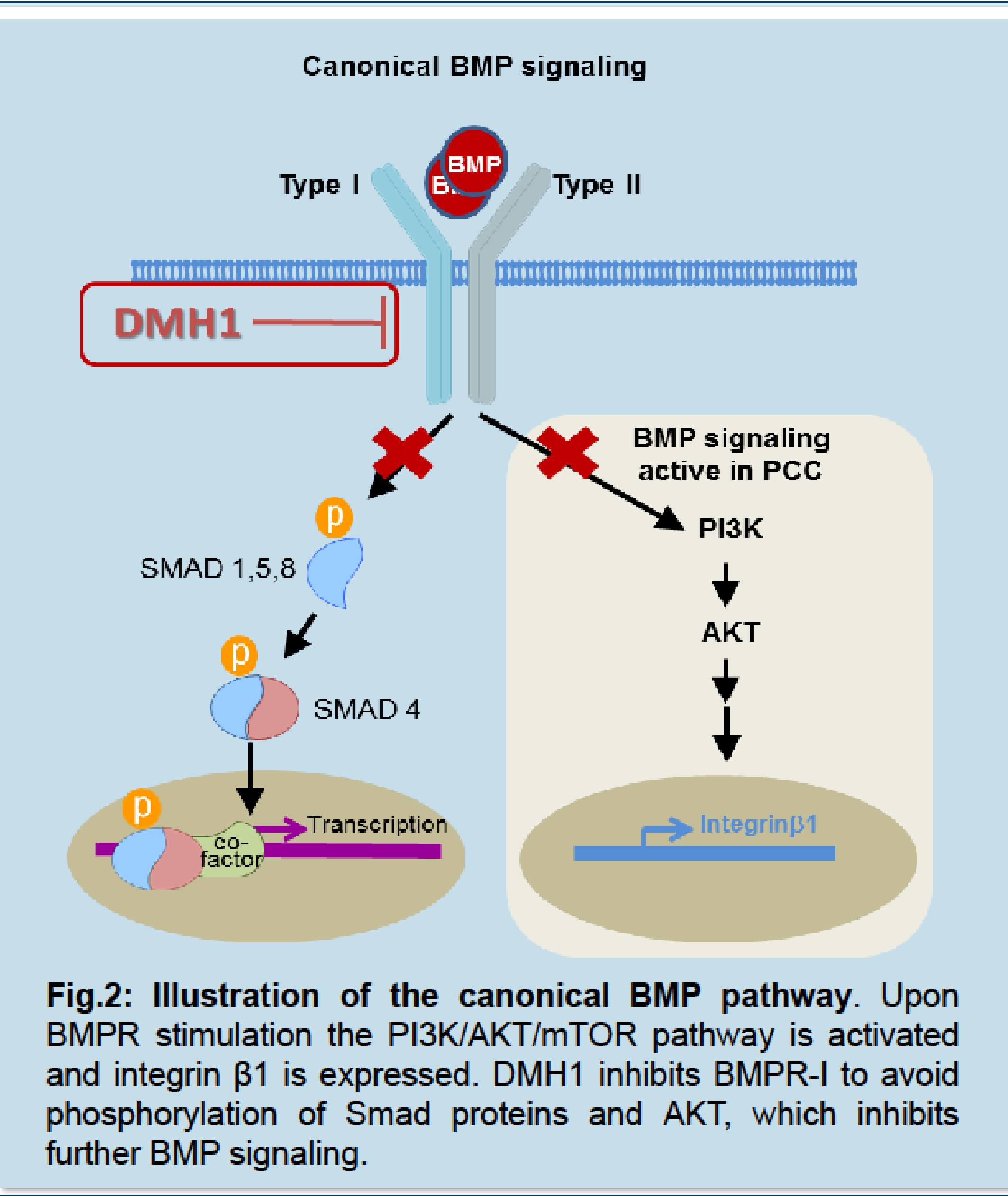

\section{Aim}

The aim of the project was to assess the role of BMP7 in PCCs of MENX rat and human PCC patients and to explore the effect of the small molecule compound, DMH1 (BMPR-I analog), in BMP7-mediated PCC tumorigenesis in vitro and ex vivo.

\section{Experimental Plan}

We used cell lines such as MPC (mouse PCC) and its aggressive derivative MTT, both with high levels of Bmp7 and primary rat PCC cells with high levels of Bmp7. To evaluate the effect of blocking Bmp7 signaling on PCC cells we treated these cells with DMH1, which selectively inhibits BMPR-I. In vitro assays assessing proliferation (MTT) and migration (Boyden chamber) were then performed. Additionally, we establishes an ex vivo system based on the rotary cell culture system from Synthecon-Cellon. With this system tissues will be cut in small pieces right after dissection of MENX rats and cultured ex vivo under DHM1 treatment.
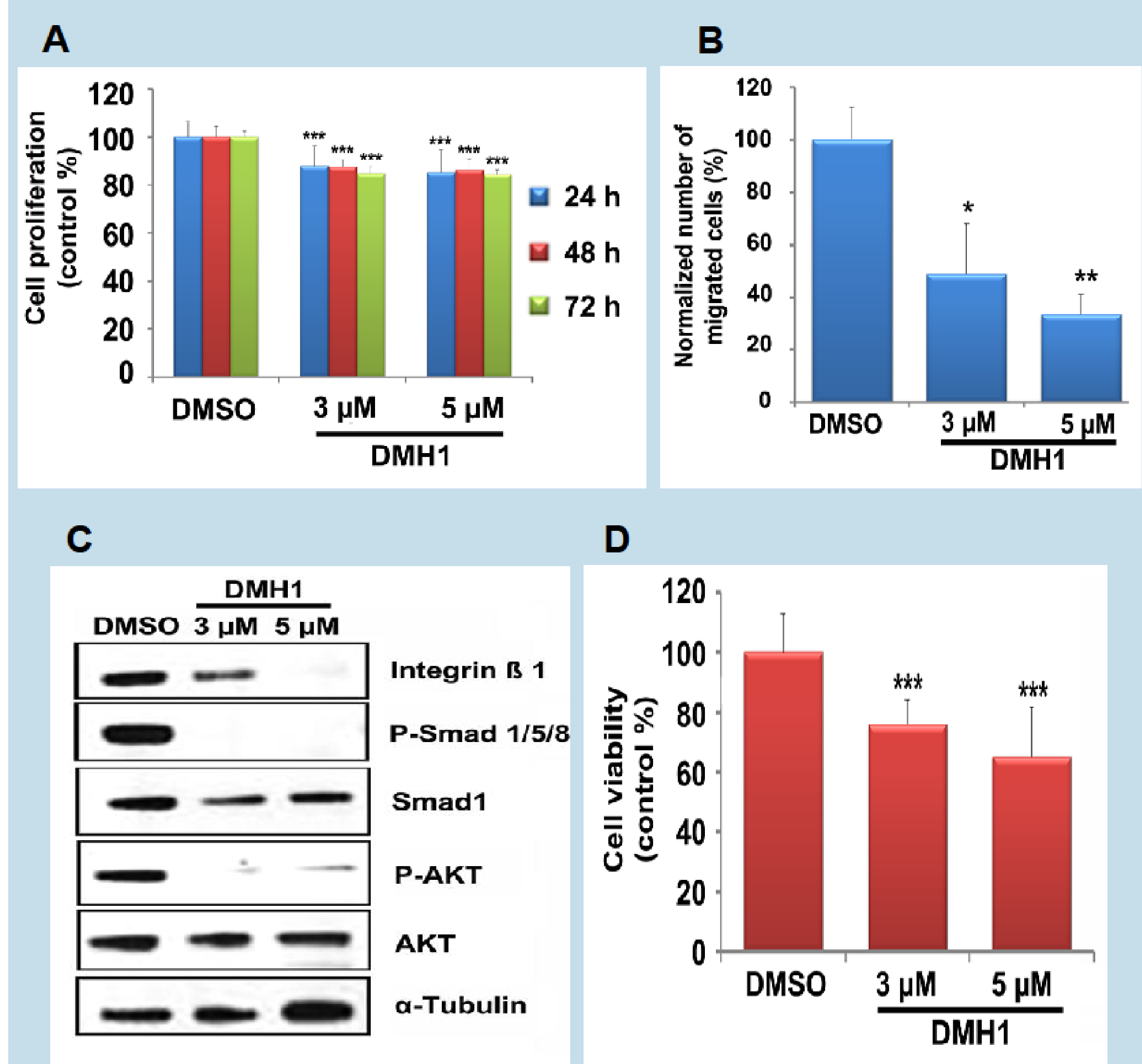

D

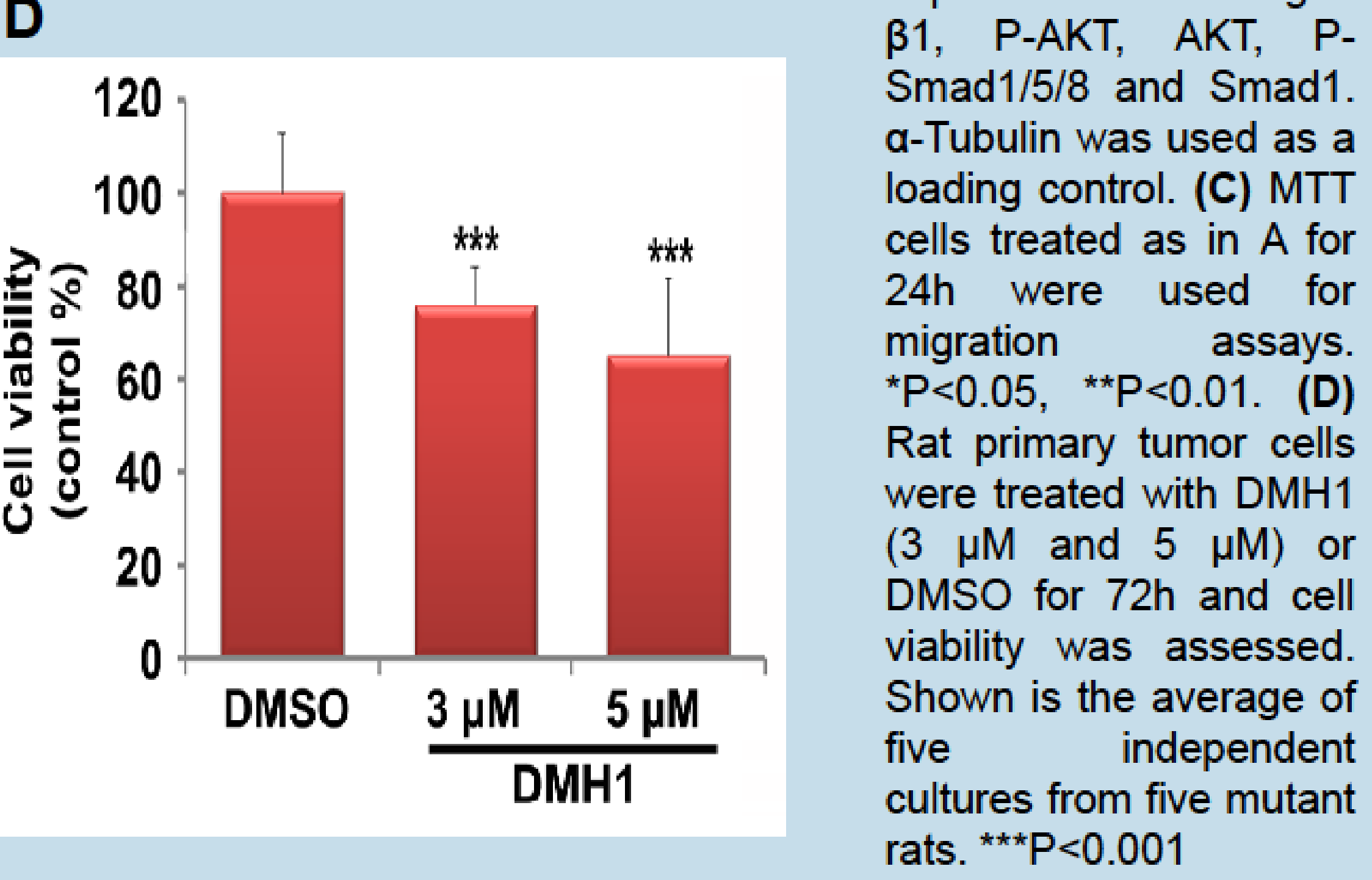

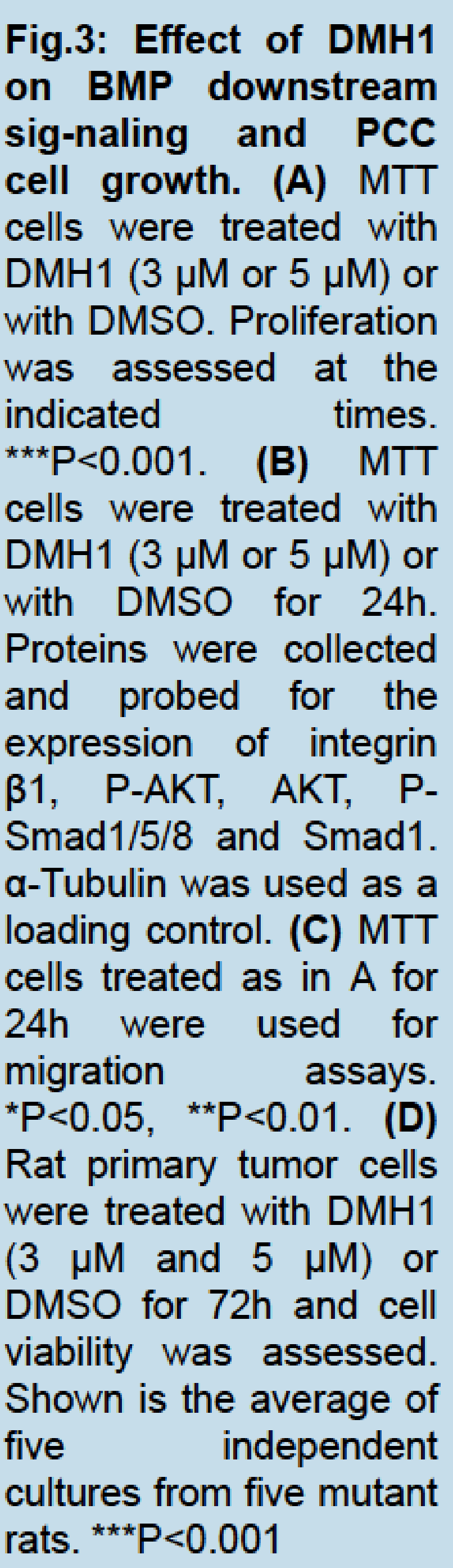

\section{Results}

The small-molecule BMP antagonist DMH1 highly selectively inhibits BMP type I receptors, but no other off-target receptors [4]. To verify whether blocking BMP receptor signaling might be a potential strategy for targeted therapy of PCC, we treated MTT cells (high endogenous Bmp7 levels) with $\mathrm{DMH} 1$ and then figures out a significantly suppressed MTT cell proliferation (Figure $3 A$ ), and even more strongly inhibited cell migration (Figure 3B). Concomitantly, we observed a dose-dependent downregulation of the expression of P-Smad1/5/8 and integrin $\beta 1$, both readouts of active BMP signaling in PCC cells, as well as of P-AKT (Figure 3C). Next, we determined a decreasing effect of $\mathrm{DMH} 1$ on rat primary PCC cells (high endogenous Bmp7 level) (Figure $3 D$ ). Furthermore we investigated the effect of DMH1 in PCC tissues of MENX affected rats ex vivo by using a rotary cell culture system. We could show a reduction of Bmp signaling downstream targets (P-AKT and P-S6) at the protein level by western blotting (reduction of around $20 \%$ ) and by IF staining (P-Smad1/5/8) (Figure 4)

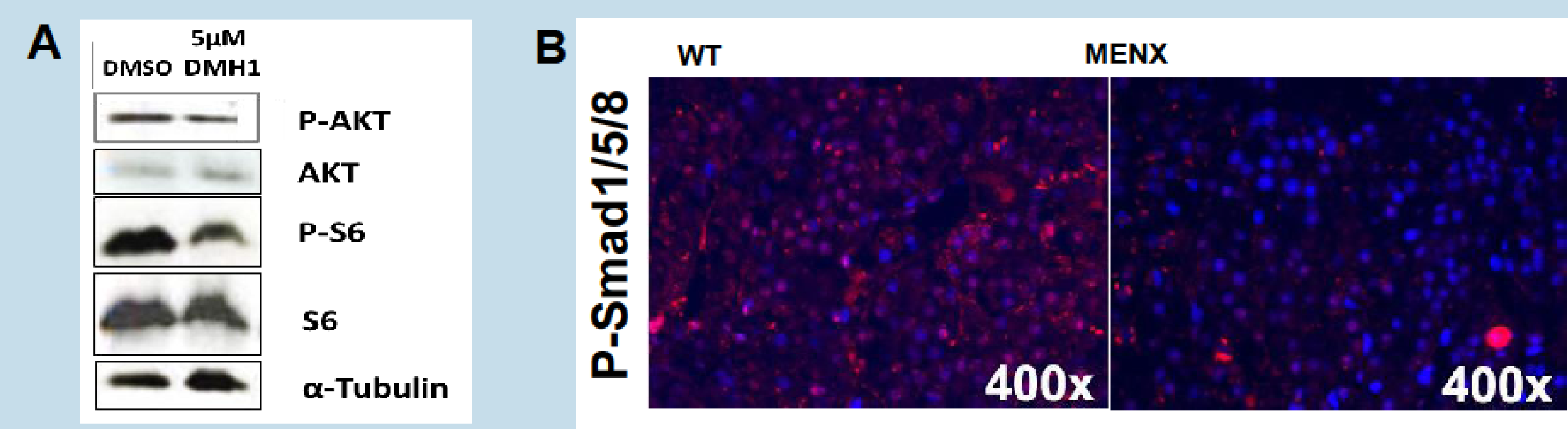

\section{Conclusions}

The Bmp pathway represents a novel therapeutic target in PCC! DMH1, a BMP receptor antagonist elicits anti-proliferative and anti-migratory responses in PCC cells with active BMP signaling in vitro and ex vivo. Future studies will address DMH1 effects on PCC in vivo.
References

[1] Pellegata, Quintanilla-Martinez et al., PNAS USA, 2006

[2] Fritz, Walch et al., Cancer Research, 2002

[3] Leinhäuser, Richter et al., Oncotarget, 2015

[4] Hao, Ho et al., Chem Biol, 2010 